\title{
Migliorare l'interazione genitore-figlio dalla nascita tramite il video feed-back: uno studio randomizzato controllato dimostra che si può
}

Roby E, Miller EB, Shaw DS, et al.

Improving Parent-Child Interactions in Pediatric Health Care: A Two-Site Randomized Controlled Trial

Pediatrics. 2021;147(3):e20201799. doi: 10.1542/peds.2020-1799

Viene studiato tramite RCT l'impatto a 6 mesi di vita di una tecnica di videofeedback (Video Interaction Project -VIP) proposta nell'ambito dei bilanci di salute in bambini di età 0-6 mesi nati da famiglie a basso reddito: si tratta di sedute di mezz'ora in cui viene videoregistrata l'interazione genitore-figlio, segue la discussione del video e la fornitura di materiale didattico appropriato al livello di sviluppo del bambino (libro, giocattolo ...) scelto per favorire l'interazione tra bambino e genitore. Sono state coinvolte $400 \mathrm{fa}$ miglie in due grandi città statunitensi randomizzate all'intervento o a ricevere le cure abituali. Nel braccio di intervento è stato osservato tramite 3 diversi questionari un aumento delle competenze cognitive e dell'interazione genitore-figlio a 6 mesi di vita. Si tratta di un interessante studio che mostra come nell'ambito delle cure primarie sia possibile migliorare le pratiche genitoriali e prevenire le disparità nello sviluppo neuropsicologico in famiglie a elevato rischio di povertà educativa.

Improving parent-child interaction from birth through a video feed-back: a randomized controlled study shows that it's feasible The impact at 6 months of life of a videofeedback technique (Video Interaction Project-VIP) proposed in the context of health assessments in children aged 0-6 months from low-income families is studied in a RCT. The half-hour sessions in which the parent-child interaction is recorded are followed by the discussion of the video and the provision of educational material appropriate to the child's development level (book, toy, ...) aimed at improving child and parent interaction. 400 families of two large US cities randomized to intervention (VIP) or to receive usual care were involved. An increase in cognitive skills and parent-child interaction was observed in the intervention arm through three different questionnaires at 6 months of age (quality of words, verbal response to parents, involvement of the parent in the advancement of the child's development). This is an interesting study that shows how in primary care it is possible to improve parenting practices and prevent disparities in neuropsychological development in families at high risk of educational poverty.

\section{Metodo}

\section{Obiettivo (con tipo studio)}

Studio randomizzato controllato in singolo cieco per valutare l'impatto a 6 mesi di vita del Programma Smart Beginnings (SB) su attività e interazione genitore-bambino, offerta alla nascita a famiglie di basso reddito.

\section{Popolazione}

Madri e bambini di famiglie a basso reddito o che rientravano nei criteri di sostegno medico arruolate in due punti nascita di New York (NYC) e Pittsburgh e randomizzate ai due gruppi di trattamento (intervento o controllo).

Criteri di inclusione:

1. neonati a termine, sani, in assenza di complicazioni mediche o interventi pre o perinatali, con previsione di ricevere assistenza pediatrica presso l'istituto;

2. il genitore è il principale caregiver o tutore legale, non affetto da patologie psichiatriche (disabilità intellettiva, schizofrenia...) o complicazioni mediche.

Criteri di esclusione: peso alla nascita $<2.500$ gr, età gestazionale $<37$ settimane, gemellarità, complicazioni mediche, trasloco in altra città in programma entro i successivi 3 anni, lingua madre del genitore/caregiver diversa da spagnolo o inglese, difficoltà di contatto, dimissione del bambino separata dalla madre, accoglienza in casa-famiglia o mancanza di stabilità abitativa, precedente partecipazione a trial similari.

\section{Intervento}

Nel gruppo di intervento sono previsti due programmi di cui solo il primo è oggetto di studio del presente trial clinico:

- Video Interaction Project 0-3 anni (VIP): 14 sessioni di 25-30 minuti somministrate nell'ambulatorio del pediatra di cure primarie in occasione dei bilanci di salute. Ogni sessione VIP prevede una breve videroregistrazione dell'interazione tra genitore e bambino, una discussione del video e la fornitura di materiale didattico appropriato al livello di sviluppo del bambino (libro, giocattolo), scelto per favorire l'interattività tra bambino e genitore. Al genitore viene fornita una copia del video e un opuscolo personalizzato con suggerimenti per svolgere poi attività.

- Family Check-Up (dai 6 mesi a 3 anni): programma di visite domiciliari in famiglie con fattori di rischio socio-economico addizionali con l'obiettivo di migliorare le pratiche genitoriali e ridurre i disturbi di comportamento dei bambini.

\section{Controllo}

Cure usuali.

\section{Outcome/Esiti}

Stimolazione cognitiva e interazione genitore/bambino misurata all'età di 6 mesi del bambino attraverso tre questionari validati e standardizzati: StimQ, Parenting Your Baby questionnaire, Parent-Child Interaction Rating Scales - Infant Adaptation (PCIRS-IA). 


\section{Tempo}

Arruolamento: Bellevue Hospital (NYC) da giugno 2015 a gennaio 2017; University of Pittsburgh Medical Center (UPMC) da giugno 2016 a ottobre 2017. Follow-up a 6 mesi di età del bambino.

\section{Risultati principali}

In totale, 403 famiglie sono state randomizzate: 200 a NYC e 203 a Pittsburgh; erano presenti molte differenze tra le popolazioni arruolate nei due centri, con una predominanza di latini a NYC (84\%) e di afroamericani a Pittsburgh (81\%). La media delle visite VIP completate è stata $3.49(\mathrm{SD}=0.97)$ su quattro possibili visite. Il numero medio di visite completato non differiva tra NYC (media $=3.40 ; \mathrm{SD}=1.13)$ e Pittsburgh $($ media $=3.58 ; \mathrm{SD}=$ $0.77, \mathrm{p}=0.17)$. La stima dell'effetto dell'intervento è stata positiva nella misurazione con il questionario StimQ2 $(d$ di Cohen $=0.28$; $\mathrm{p}=0.01)$; e nelle varie sottocomponenti: StimQ2 READ $(d=0.23$; $\mathrm{p}=0.02)$, qualità delle parole $(d=0.26 ; \mathrm{p}=0.01)$, risposta verbale ai genitori $(d=0.21 ; \mathrm{p}=0.04)$, coinvolgimento del genitore nell'avanzamento dello sviluppo del figlio $(d=0.25 ; \mathrm{p}=0.01)$. Nel Parent-Child Interaction Rating Scales - Infant Adaptation la stimolazione cognitiva è risultata aumentata $(d=0.40 ; \mathrm{p}<0.001)$ così come i domini indicanti il supporto per lo sviluppo cognitivo $(d=0.36 ; \mathrm{p}<0.001)$, la quantità $(d=0.40 ; \mathrm{p}<0.001)$ e la qualità di linguaggio $(d=0.37 ; \mathrm{p}<0.001)$.

\section{Conclusioni}

SB è uno dei primi modelli a step che collega e integra gli interventi basati sull'evidenza nella pediatria delle cure primarie a visite domiciliari con funzione di prevenire le disparità sullo sviluppo neuropsicologico e la preparazione scolastica in famiglie a elevato rischio di povertà educativa. Questo studio ha rilevato che VIP come primo pezzo di SB impatta sui comportamenti genitoriali anche in aree geografiche distanti e con genitori di etnia e caratteristiche demografiche diverse.

\section{Altri studi sull'argomento}

Poiché in questo numero delle Pagine elettroniche di Quaderni acp presentiamo un altro intervento tramite video feedback presentiamo gli altri studi sull'argomento in una sola scheda newsletter. Pertanto il lettore troverà le revisioni sistematiche sugli interventi per sostenere il parenting nelle cure primarie pediatriche e sugli interventi di video feedback all'interno della scheda newsletter Ridurre i problemi comportamentali nei bambini piccoli attraverso il video-feedback. Trial clinico randomizzato pragmatico.

\section{Che cosa aggiunge questo studio}

Conferma l'efficacia di un intervento universale attraverso la metodica del video feedback eseguito in ambulatorio all'interno delle cure primarie pediatriche in due aree cittadine a basso reddito in USA.

\section{Commento}

\section{Validità interna}

Disegno dello studio: complessivamente considerato di buona qualità metodologica. Non viene esplicitata la modalità di randomizzazione. Degli eleggibili circa $2 / 3$ non entrano nello studio; gli autori descrivono accuratamente le motivazioni del mancato arruolamento. Le popolazioni arruolate nei due siti presentano diverse differenze demografiche. Sebbene sia stata valutata a priori la potenza dello studio e sia stato raggiunto il numero scelto di 400 famiglie, lo studio non è stato disegnato per poter valutare differenze in sottogruppi di popolazione all'interno dello stesso sito ma solo tra i due siti. La cecità, che ha riguardato solo i ricercatori che hanno effettuato l'analisi dei risultati, è adeguata per il tipo di studio. Viene ricercata la fidelity al trattamento ma non viene verificata. La partecipazione al trattamento dalla nascita ai 6 mesi è stata elevata. La perdita al follow-up bassa e bilanciata tra i gruppi: $10 \%$. Le famiglie hanno ricevuto un piccolo incentivo in denaro (50\$) per la partecipazione al controllo dei 6 mesi. L'analisi dei risultati è stata condotta per intention to treat. Non è stata svolta una analisi per fattori confondenti. Per valutare la dimensione dell'effetto viene valutata la $\mathrm{d}$ di Cohen (effetto molto piccolo $d<0.20$; piccolo $d$ da 0.20 a 0.50 ; medio $d$ da 0.50 a 0.80 ; grande $d>0.80$ ). Non vengono analizzati sottogruppi per valutare l'effetto di determinate variabili quali il livello educativo dei genitori, il livello di povertà, primi figli vs secondi-terzogeniti sugli esiti dell'intervento. Nonostante gli autori presentino l'intervento Family Check-Up, questo non è stato mai utilizzato a causa del breve periodo di follow-up (sei mesi su tre anni di intervento previsto dal protocollo). E possibile che l'inclusione dei soli neonati sani a termine abbia a priori escluso bambini con alto rischio di povertà ed emarginazione (ad esempio bambini nati da madri con dipendenza da alcool o droga, bambini in cui la prematurità stessa può essere il frutto di povertà materna, gravidanza poco seguite, scarso accesso ai servizi, etc). Il protocollo di studio è stato registrato ed è disponibile su www.clinicaltrials. gov (NCT02459327).

Esiti: sono clinicamente rilevanti e ben definiti. L'effetto dell'intervento complessivamente stimato con la $d$ di Cohen varia da 0.20 a 0.40 in linea con quanto misurato in precedenza con interventi preventivi più intensivi (es. programmi di home visiting).

Conflitto di interesse: gli autori dichiarano assenza di conflitto di interesse.

\section{Trasferibilità}

Popolazione studiata: l'intervento è destinato a famiglie a maggior rischio di povertà educativa. Le condizioni di svantaggio sociale, rete di sostegno e sistema di welfare sono differenti tra la nostra realtà e quella statunitense.

Tipo di intervento: il Video Interaction Project è proposto da un operatore specificamente addestrato; mentre il Family Check-Up è un programma di visite domiciliari; ambedue questi interventi possono essere realizzati anche nella nostra realtà formando gli operatori e organizzando la rete dei professionisti per le visite domiciliari. 


\section{Scheda redatta dal gruppo di lettura di Reggio Emilia:}

Francesca Bontempo, Carlo Boni, Jennifer Chiarolanza, Angelo Cigarini, Elena Corbelli, Annalisa Correggi, Anna Maria Davoli, Anna Rita Di Buono, Elena Ferrari, Monica Malventano, Elena Manzotti, Maddalena Marchesi, Gino Montagna, Luciana Monti, Rosaria Ollari, Luisa Seletti, Mariassunta Torricelli, Maria Candida Tripodi, Daniela Vignali, Marialuisa Villani, Costantino Pan$z a$.

\section{Glossario}

\section{$d$ di Cohen}

La $\boldsymbol{d}$ di Cohen è una misura della dimensione dell'effetto che, insieme ai parametri aggiuntivi del livello di significatività e potenza statistica, è utilizzata per stimare le dimensioni del campione per i test statistici, indica la differenza standardizzata tra due medie, cioè la forza della relazione tra due variabili con una misura di effetto assoluta.

\section{La Newsletter Pediatrica}

La Newsletter Pediatrica nasce come bollettino di aggiornamento scientifico-bibliografico online dell'ACP e di Quaderni acp e da novembre 2015 costituisce una rubrica fissa delle Pagine elettroniche.

Le riviste monitorate sono le seguenti:

- $\quad$ Lancet

- $\quad$ British Medical Journal (BMJ)

- Journal of American Medical Association (JAMA)

- New England Journal of Medicine

- Archives of Diseases in Childhood (ADC)

- Paediatrics

- Journal of Pediatrics

- JAMA Pediatrics

- Cochrane Database of Systematic Reviews

- $\quad$ BMC pediatrics

- $\quad$ BMJUpdates

Attraverso questo lavoro di screening vengono identificati gli articoli potenzialmente rilevanti che sono quindi letti in maniera approfondita, valutati rispetto alla loro qualità metodologica, presentati e discussi nel corso di riunioni mensili. Lo studio selezionato per essere recensito nella newsletter viene riassunto mettendo in evidenza le sue principali caratteristiche (obiettivo, popolazione, intervento, esiti, risultati e conclusioni degli autori) e viene accompagnato da commenti per:

- collocarlo nel panorama più ampio della letteratura scientifica già disponibile sull'argomento, per conferire il giusto peso alle nuove informazioni prodotte e verificarne la coerenza con il patrimonio di conoscenze accumulato;

- discuterne gli aspetti metodologici (qualità, esiti considerati, validità interna);

- $\quad$ evidenziare il possibile impatto sulla pratica clinica (validità esterna). 